\title{
RELATIVISTIC PSEUDOSPIN SYMMETRY AND THE STRUCTURE OF NUCLEAR STATES
}

\author{
A. LEVIATAN ${ }^{1}$ AND J.N. GINOCCHIO ${ }^{2}$ \\ ${ }^{1}$ Racah Institute of Physics, The Hebrew University, \\ Jerusalem 91904, Israel \\ 2 Theoretical Division, Los Alamos National Laboratory, \\ Los Alamos, New Mexico 87545, USA
}

\section{Introduction}

The concept of pseudospin symmetry $[1,2]$ is based on the empirical observation of quasi-degenerate pairs of certain normal-parity shell-model orbitals with non-relativistic quantum numbers

$$
\left(n_{r}, \ell, j=\ell+\frac{1}{2}\right) \text { and }\left(n_{r}-1, \ell+2, j=\ell+\frac{3}{2}\right) .
$$

Here $n_{r}, \ell$, and $j$ are the single-nucleon radial, orbital, and total angular momentum quantum numbers, respectively. The doublet structure, is expressed in terms of a "pseudo" orbital angular momentum $\tilde{\ell}=\ell+1$ and "pseudo" spin, $\tilde{s}=1 / 2$, coupled to $j=\tilde{\ell} \pm \tilde{s}$. For example, $\left(2 p_{3 / 2}, 1 f_{5 / 2}\right)$ will have $\tilde{\ell}=2$, etc. This pseudospin symmetry plays a central role in nuclei [3] and only recently has it been shown to originate from a relativistic symmetry of the Dirac Hamiltonian [4, 5]. The members of the pseudospin doublet exhibit the following features. (i) They have different angular momentum quantum numbers $(\ell, j) \leftrightarrow(\ell+2, j+1)$ for states with aligned/unaligned spin. (ii) They have different radial wave functions and, in particular, different number of nodes $n_{r} \leftrightarrow n_{r}-1$. (iii) They involve only normal-parity shell-model orbitals. The "intruder" levels with aligned spin and no nodes, e.g. $0 g_{9 / 2}, 0 h_{11 / 2}, 0 i_{13 / 2}$ do not form quasi-degenerate doublets. In the present contribution we show that a natural explanation for all these features can be obtained by combining the relativistic attributes of pseudospin symmetry with known properties of Dirac bound states [6]. 


\section{Dirac Wave Functions in Central Fields}

The eigenstates of a spherically symmetric Dirac Hamiltonian have the form $\Psi_{\kappa, m}=\left(\frac{G_{\kappa}}{r}\left[Y_{\ell} \chi\right]_{m}^{(j)}, i \frac{F_{\kappa}}{r}\left[Y_{\ell^{\prime}} \chi\right]_{m}^{(j)}\right)$ where $G_{\kappa}(r)$ and $F_{\kappa}(r)$ are the radial wave functions of the upper and lower components respectively. The labels $\kappa=\mp(j+1 / 2)$ and $\ell^{\prime}=\ell \pm 1$ for $j=\ell \pm 1 / 2$. As bound state solutions, both $G(r)$ and $F(r)$ vanish at $r=0$ and $r=\infty$ and the binding energy is positive $(M-E)>0$. Further properties of these wave functions can be inferred from the following equation for the product $G F$

$$
(G F)^{\prime}=A(r) F^{2}-B(r) G^{2},
$$

where $A(r)=\left[E+M+V_{S}(r)-V_{V}(r)\right], B(r)=\left[E-M-V_{S}(r)-V_{V}(r)\right]$. For relativistic mean fields relevant to nuclei, the scalar potential, $V_{S}(r)$, is attractive and the vector potential, $V_{V}(r)$, is repulsive. For typical values $A(r)>0$, while $B(r)$ changes monotonically from $B(0)>0$ to $B(\infty)=$ $(E-M)<0$. Since $G F$ vanishes at both end points. we find by Eq. (2) that it is an increasing negative function at large $r$, while at small $r, G F$ is a decreasing negative function for $\kappa<0$ and an increasing positive function for $\kappa>0$. Futhermore, since $B(r)>0$ at the nodes of $F$ and $G$ (nodes can occur only in the region where the kinetic energy is positive), it follows that $G F$ is a decreasing function at the nodes of $F$, and an increasing function at the nodes of $G$. Exploiting all these properties, we observe that for $\kappa>0$, $G F$ is positive at small $r$ and negative at large $r$, and hence has an odd number of zeroes. The first and last zeroes of $G F$ correspond to nodes of $F$ and since the nodes of $F$ and $G$ alternate [6,7], then the number of nodes of $F$ exceed by one the number of nodes of $G$. On the other hand, for $\kappa<0$, $G F$ has the same (negative) sign near both end points, and hence has an even number of zeroes. By similar arguments we find that in this case the first and last zeroes of $G F$ are nodes of $G$ and $F$ respectively, and that $G$ and $F$ have the same number of nodes. These properties of the radial wave functions are illustrated in Figs. 1a-d for the $2 p_{3 / 2}(\kappa=-2), 1 f_{5 / 2}(\kappa=3)$ states. Altogether we have,

$$
\begin{array}{ll}
\kappa<0: & n_{F}=n_{G} \\
\kappa>0: & n_{F}=n_{G}+1
\end{array}
$$

where $n_{F}$ and $n_{G}$ denote the number of internal nodes of $F$ and $G$.

When the radial parts of the wave functions $G$ and $F$ have no nodes $\left(n_{F}=n_{G}=0\right)$, the corresponding bound states can appear only in the $j=\ell+1 / 2$ state $(\kappa<0)$, but not in the $j=\ell-1 / 2$ state $(\kappa>0)[6,8]$. The reason being that in this case $G F$ has the same sign for all $r$ and since always $G F<0$ at large $r$, then it is also negative at small $r$ and therefore 

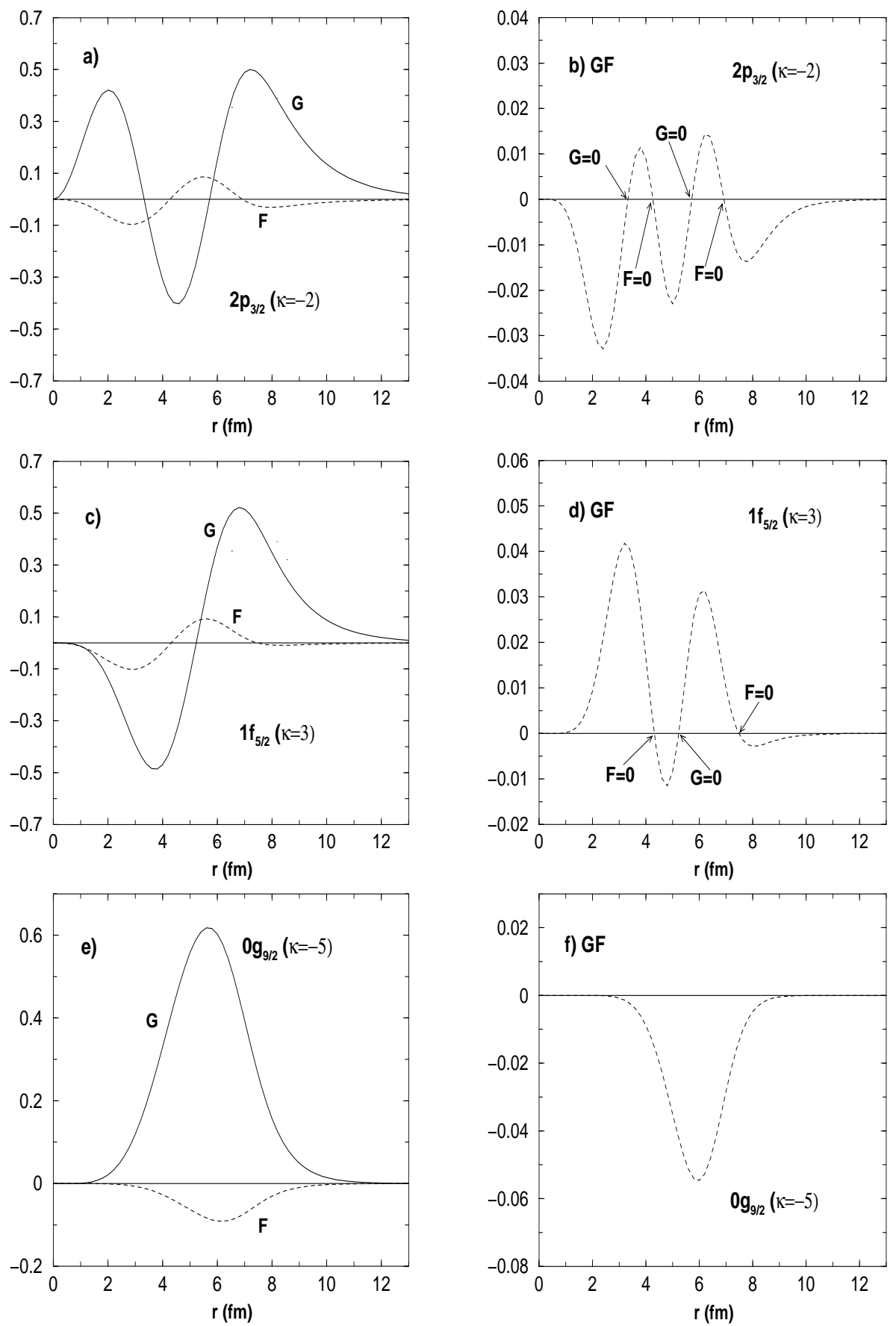

Figure 1. (a) The radial upper component $(G)$ and lower component $(F)$ in $(\text { Fermi })^{-1 / 2}$ and (b) the corresponding product $G F$ in $(\text { Fermi })^{-1}$ of the $2 p_{3 / 2}(\kappa=-2)$ state. (c) and (d) The same for the $1 f_{5 / 2}(\kappa=3)$ state. (e) and (f) The same for the $0 g_{9 / 2}(\kappa=-5)$ state. All states are eigenstates of a Dirac Hamiltonian with scalar $(S)$ and vector $(V)$ potentials: $V_{S, V}(r)=\alpha_{S, V}\left[1+\exp \left(\frac{r-R}{a}\right)\right]^{-1}$ with parameters: $\alpha_{S}=-358, \alpha_{V}=292$ $\mathrm{MeV}, R=7 \mathrm{fm}, a=0.6 \mathrm{fm}$, tuned to the neutron spectra of ${ }^{208} \mathrm{~Pb}$. Taken from Ref. [6]. 
must have $\kappa<0$. An example is shown in Figs. 1e-f for the case of the $0 g_{9 / 2}$ $(\kappa=-5)$ intruder state.

Finally, we note that for bound states to exist, there must be a region where $B(r)>0$. This is due to the fact that, as mentioned, $G F$ is an increasing negative function at large $r$, and to enable it to vanish at $r=0$, its derivative $(G F)^{\prime}$ must change sign from positive to negative in some region. A glance at Eq. (2) shows that since $A(r)>0$, a necessary (but not sufficient) condition for $(G F)^{\prime}$ to become negative is that

$$
B(r)=\left[E-M-V_{S}(r)-V_{V}(r)\right]>0 \text { for some } \mathrm{r} .
$$

The above condition means that in order that bound states exist, there has to be a region where the depth of the average attractive single-nucleon potential, $V_{S}(r)+V_{V}(r)$, is larger than the binding energy.

\section{Relativistic Pseudospin Symmetry}

All of the above results are relevant for understanding properties of states in the relativistic pseudospin scheme $[4,5]$. When $V_{S}=-V_{V}$, the Dirac Hamiltonian is a scalar under an $\mathrm{SU}(2)$ algebra, $\left[H, \hat{\tilde{S}}_{\mu}\right]=0$. If in addition the potentials are spherically symmetric, the Dirac Hamiltonian has an additional invariant $\mathrm{SU}(2)$ algebra, $\left[H, \hat{\tilde{L}}_{\mu}\right]=0$. The relativistic pseudospin generators, $\hat{\tilde{S}}_{\mu}$, and relativistic pseudo-orbital angular momentum operators, $\hat{\tilde{L}}_{\mu}$, are given by

$$
\hat{\tilde{S}}_{\mu}=\left(\begin{array}{cc}
\hat{\tilde{s}}_{\mu} & 0 \\
0 & \hat{s}_{\mu}
\end{array}\right) \quad, \quad \hat{\tilde{L}}_{\mu}=\left(\begin{array}{cc}
\hat{\tilde{\ell}}_{\mu} & 0 \\
0 & \hat{\ell}_{\mu}
\end{array}\right) .
$$

Here $\hat{\tilde{s}}_{\mu}=U_{p} \hat{s}_{\mu} U_{p}$ and $\hat{\tilde{\ell}}_{\mu}=U_{p} \hat{\ell}_{\mu} U_{p}$, where $\hat{s}_{\mu}=\sigma_{\mu} / 2$ and $\hat{\ell}_{\mu}=\boldsymbol{r} \times \boldsymbol{p}$ are the usual spin and orbital angular momentum operators respectively, $\sigma_{\mu}$ the Pauli matrices and $U_{p}=\frac{\boldsymbol{\sigma} \cdot \boldsymbol{p}}{p}$ is the momentum-helicity unitary operator introduced in [9]. The sets $\left\{\hat{\tilde{S}}_{\mu}, \hat{\tilde{s}}_{\mu}, \hat{s}_{\mu}\right\}$ and $\left\{\hat{\tilde{L}}_{\mu}, \hat{\tilde{\ell}}_{\mu}, \hat{\ell}_{\mu}\right\}$ form two triads of $S U(2)$ algebras. The $\hat{\tilde{S}}_{\mu}$ and $\hat{\tilde{L}}_{\mu}$ operators act on the four-components Dirac wave functions. The $\hat{\tilde{s}}_{\mu}$ and $\hat{\tilde{l}}_{\mu}$ operators form the non-relativistic pseudospin and pseudo-orbital angular momentum algebras respectively, and act on the upper components of the Dirac wave functions. The $\hat{s}_{\mu}$ and $\hat{\ell}_{\mu}$ act on the "small" lower components of the Dirac wave functions. The pseudospin $\tilde{s}=1 / 2$ and pseudo-orbital angular momentum $\tilde{\ell}$ are seen from Eq. (5) to be the ordinary spin and ordinary orbital angular momentum 
of the lower component of the Dirac wave functions. The two states in the pseudospin doublet share a common pseudo-orbital angular momentum $\tilde{\ell}$, which is coupled to a pseudospin $\tilde{s}=1 / 2$, and thus have the form

$$
\begin{aligned}
\Psi_{\kappa<0, m} & =\left(\frac{G_{\kappa<0}(r)}{r}\left[Y_{\tilde{\ell}-1} \chi\right]_{m}^{(j)}, i \frac{F_{\kappa<0}(r)}{r}\left[Y_{\tilde{\ell}} \chi\right]_{m}^{(j)}\right) \quad j=\tilde{\ell}-\frac{1}{2} \\
\Psi_{\kappa^{\prime}>0, m} & =\left(\frac{G_{\kappa^{\prime}>0}(r)}{r}\left[Y_{\tilde{\ell}+1} \chi\right]_{m}^{\left(j^{\prime}\right)}, i \frac{F_{\kappa^{\prime}>0}(r)}{r}\left[Y_{\tilde{\ell}} \chi\right]_{m}^{\left(j^{\prime}\right)}\right) j^{\prime}=\tilde{\ell}+\frac{1}{2} .
\end{aligned}
$$

The underlying Dirac structure ensures that the wave function of the upper component of the Dirac eigenfunction has a spherical harmonic of rank $\ell=\tilde{\ell}-1$ for aligned spin: $j=\tilde{\ell}-1 / 2=\ell+1 / 2$, and a spherical harmonic of $\operatorname{rank} \ell+2=\tilde{\ell}+1$ for unaligned spin: $j^{\prime}=\tilde{\ell}+1 / 2=(\ell+2)-1 / 2$. This explains the particular angular momenta defining the pseudospin doublet of Eq. (1). In the pseudospin symmetry limit the two states in Eq. (6) form a degenerate doublet $(S=1 / 2)$, and are connected by the pseudospin generators $\hat{\tilde{S}}_{\mu}$ of Eq. (5). The corresponding upper components are a doublet with respect to the set $\hat{\tilde{s}}_{\mu}$ (the non-relativistic pseudospin algebra). Since the latter, by definition, intertwine space and spin, they can connect states for which the upper components have different radial wave functions, $G_{\kappa<0}(r) \neq G_{\kappa^{\prime}>0}(r)$. On the other hand, the corresponding lower components are a doublet with respect to the ordinary spin $\hat{s}_{\mu}$, and hence, in the pseudospin limit, their radial wave functions are equal up to a phase,

$$
F_{\kappa<0}(r)=F_{\kappa^{\prime}>0}(r) \text {. }
$$

In particular, $F_{\kappa<0}(r)$ and $F_{\kappa^{\prime}>0}(r)$ have the same number of nodes, which we denote by $n_{r}$. If we now use the result of Eq. (3), we find for $n_{r} \neq 0$ that $G_{\kappa<0}(r)$ in Eq. (6) has also $n_{r}$ radial nodes, while $G_{\kappa^{\prime}>0}(r)$ has $n_{r}-1$ nodes. This explains the structure of nodes in the pseudospin doublet of Eq. (1). The simple relation in Eq. (7) between the radial wave functions of the lower components of the two states in the doublet, dictates this particular relation between the radial nodes of the corresponding upper components. This result cannot be obtained if one considers just the non-relativistic pseudospin algebra, $\hat{\tilde{s}}_{\mu}$, and is a direct outcome of the behavior of nodes of Dirac bound states and the identification of pseudospin as a relativistic symmetry of the Dirac Hamiltonian.

As we have shown, bound Dirac states, for which both the upper and lower components have no nodes $\left(n_{r}=0\right)$ can occur only for $\kappa<0$ and not for $\kappa^{\prime}>0$. From Eq. (6) we find that such states have pseudo-orbital angular momentum $\tilde{\ell}$ and total angular momentum $j=\tilde{\ell}-1 / 2$. As mentioned, these intruder states are ignored in the non-relativistic pseudospin scheme, and it is only the relativistic interpretation of pseudospin symmetry, combined 
with known properties of Dirac bound states, which enable a classification for these states, as well as provide a natural explanation why these states do not have a pseudospin partner which is an eigenstate of the Hamiltonian.

The exact pseudospin limit requires that $V_{S}(r)=-V_{V}(r)$, which implies that $B(r)=E-M$. It is clear that under such circumstances the condition

of Eq. (4) cannot be fulfilled for bound states with positive binding energy $M-E>0$. This explains why in the exact pseudospin limit, there are no bound Dirac states and, therefore, by necessity the pseudospin symmetry must be broken in nuclei. Nevertheless, a variety of realistic mean field calculations show that the required breaking of pseudospin symmetry in nuclei is small $[10,11,12,13]$. Quasi-degenerate doublets of normal-parity states, and abnormal-parity levels without a partner eigenstate persist in the spectra, and the relation of Eq. (7) is obeyed to a good approximation, especially for doublets near the Fermi surface. As discussed, these features are sufficient to ensure the observed structure of nodes occurring in pseudospin doublets and the special status of intruder levels in nuclei.

\section{Acknowledgements}

It is a pleasure to dedicate this article to P. Ring on the occasion of his 60th birthday. This research was supported in part by the U.S.-Israel Binational Science Foundation and in part by the United States Department of Energy under contract W-7405-ENG-36.

\section{References}

1. A. Arima, M. Harvey and K. Shimizu, Phys. Lett. B 30 (1969) 517.

2. K.T. Hecht and A. Adler, Nucl. Phys. A 137 (1969) 129.

3. J.N. Ginocchio and A. Leviatan, contribution to these proceedings and references therein.

4. J.N. Ginocchio, Phys. Rev. Lett. 78 (1997) 436.

5. J.N. Ginocchio and A. Leviatan, Phys. Lett. B 425 (1998) 1.

6. A. Leviatan and J.N. Ginocchio, submitted to Phys. Lett. B, nucl-th/0108016.

7. M.E. Rose and R.R. Newton, Phys. Rev. 82 (1951) 470.

8. M. Hirooka, G. Konisi and T. Satto, Prog. Theor. Phys. 41 (1969) 161.

9. A. L. Blokhin, C. Bahri, and J.P. Draayer, Phys. Rev. Lett. 74 (1995) 4149.

10. J.N. Ginocchio and D. G. Madland, Phys. Rev. C 57 (1998) 1167.

11. G.A. Lalazissis, Y.K. Gambhir, J.P. Maharana, C.S. Warke and P. Ring, Phys. Rev. C 58 (1998) R45.

12. J. Meng, K. Sugawara-Tanabe, S. Yamaji, P. Ring and A. Arima, Phys. Rev. C 58 (1998) R628; J. Meng, K. Sugawara-Tanabe, S. Yamaji and A. Arima, Phys. Rev. C 59 (1999) 154.

13. J.N. Ginocchio and A. Leviatan, Phys. Rev. Lett. 87 (2001) 072502. 